\title{
Radiation From Slots on Dielectric-Clad and Corrugated Cylinders
}

\author{
James R. Wait and Alyce M. Conda
}

\begin{abstract}
An approximate formula is derived for the radiation pattern of an axially slotted cylinder with a thin dielectric coating. The accuracy of the formula is shown to be sufficient for practical purposes. Using a similar method, the pattern function for a slot on a corrugated cylinder is derived. Extensive numerical results are presented for both dielectricclad and corrugated cylinders.
\end{abstract}

\section{Introduction}

The radiation from slots on smooth circular cylinders is now well understood. However, if the cylindrical surface is coated with a dielectric film, the patterns can be appreciably modified. Although a rigorous expression can be derived for the fields of an arbitrary slot on a circular cylinder with a dielectric coating, the resulting formulas are very cumbersome. ${ }^{1}$ For this reason, it seems worthwhile to modify the final expressions for the field by making. several approximations on the basis that the thickness of the dielectric film is small. Furthermore, it is of interest to consider the problem by using approximate boundary conditions. This latter method can also be used to treat the corrugated cylinder under the restriction that the teeth are closely spaced with respect to the wavelength.

An attempt will be made in the present paper to investigate the accuracy of the simplifying approximations for dielectric-clad cylinders. A comparison is also made between thin dielectric coatings and corrugations on an axially slotted circular cylinder, from the standpoint of the directionality of the radiation.

\section{Dielectric-Clad Cylinder}

The cylinder is taken to be of radius $a$ and the concentric dielectric coating has a radius $b$, as indicated in figure $1 \mathrm{a}$ and $1 \mathrm{~b}$. Cylindrical coordinates $(\rho, \phi, z)$ are chosen to be coaxial with the cylinder. The electrical constants of the coating are $\epsilon$ and $\mu$, and those of the homogeneous (air) space outside are $\epsilon_{0}$ and $\mu_{0}$. The tangential electric fields on the slot are to be specified. In the case of a thin axial slot (where the slot is parallel to the cylinder axis), the electric field, $E$, in the equatorial plane is vertically polarized, possessing only a $z$ component. It was shown before that (see footnote 1)

$$
E_{\phi}=\left[\frac{k_{0}}{2 \pi^{2}} \int_{z_{1}}^{z_{2}} V\left(z^{\prime}\right) d z^{\prime}\right] \frac{e^{-i k_{0 \rho} \rho}}{\rho} P(\phi)
$$

where $k_{0}=\left(\epsilon_{0} \mu_{0}\right)^{\frac{1}{2} \omega}=2 \pi /$ free space wavelength, and where $V\left(z^{\prime}\right)$ is the transverse voltage along the slot

$1 \mathrm{~J} . \mathrm{R}$. Wait and W. E. Mientka, Slotted-cylinder antenna with a dielectric coating, J. Research NBS 58, 287 (1957) RP2762.

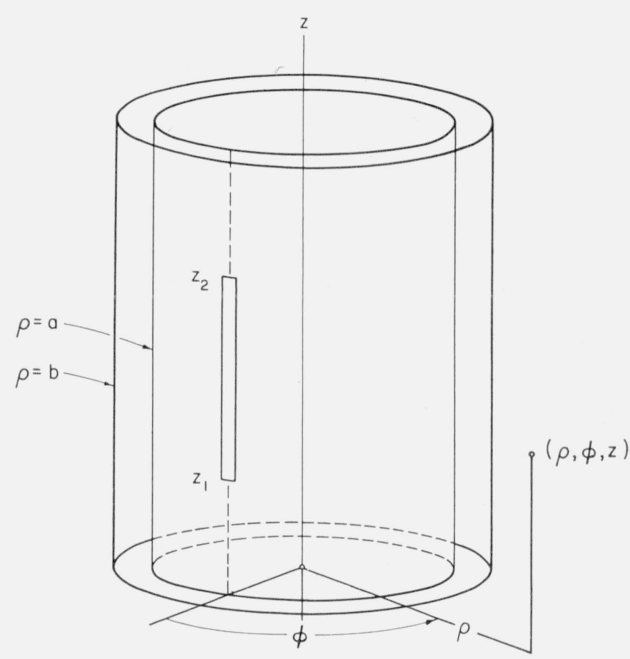

Figure 1a. Axial slot on a metallic circular cylinder of infinite length with a dielectric coating.

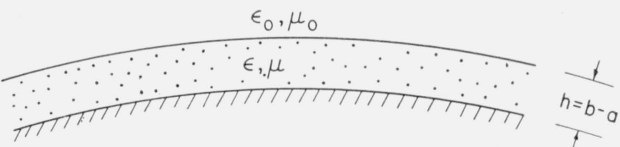

Figure 1b. Sectional view of the dielectric coating.

that extends from $z=z_{1}$ to $z_{2}$ at $\rho=a$ and $\phi=0$. The pattern factor that characterizes the azimuthal variation of the radiated field is given by

$$
P(\phi)=\frac{2 i}{\pi(k b)\left(k_{0} a\right)} \sum_{m=0}^{\infty} \frac{\epsilon_{m} \cos m \phi e^{i m \pi / 2}}{\left(\frac{k_{0} \mu}{k \mu_{0}}\right) H_{m}\left(k_{0} b\right) T_{m}-H_{m}^{\prime}\left(k_{0} b\right) L_{m}}
$$

where

$$
\begin{aligned}
& T_{m}=J_{m}^{\prime}(k b) H_{m}^{\prime}(k a)-J_{m}^{\prime}(k a) H_{m}^{\prime}(k b), \\
& L_{m}=J_{m}(k b) H_{m}^{\prime}(k a)-J_{m}^{\prime}(k a) H_{m}(k b),
\end{aligned}
$$

$k=(\epsilon \mu)^{1 / 2} \omega$, and $J_{m}$ and $H_{m}$ are the Bessel function and the Hankel function of the second kind, of order $m$, respectively. The prime indicates the derivative with respect to the argument of the 
function. When the slot is of infinite length, the radiated field is a cylindrical wave ${ }^{2}$ and the field is given by

$$
E_{\phi}=-i 60 \epsilon_{0} \omega V\left(\frac{2}{\pi k_{0} \rho}\right)^{1 / 2} e^{-i\left(k_{0} \rho-\pi / 4\right)} P(\phi)
$$

which has the same azimuthal dependence as the spherical wave radiated from a finite slot.

Some numerical values for the function $P(\phi)$ were given in a previous paper (see footnote 1 ). The cases considered were for $k a=2$ and 3 , and various values of $b / a, \epsilon / \epsilon_{0}$ and $\mu / \mu_{0}$. The numerical work involved is extensive. In view of the complexity of the above formula for $P(\phi)$, an effort was made to simplify it by making several judicious approximations. The validity of this procedure is best checked by comparing the numerical values computed from the approximate and the exact formulas. This work will be described in what follows.

Noting the Taylor expansions

$$
\begin{aligned}
& J_{m}^{\prime}(k a)=J_{m}^{\prime}(k b)+(k a-k b) J_{m}^{\prime \prime}(k b)+\ldots ., \\
& H_{m}^{\prime}(k a)=H_{m}^{\prime}(k b)+(k a-k b) H_{m}^{\prime \prime}(k b)+\ldots .,
\end{aligned}
$$

it follows that to a first order in $k(b-a)$,

$$
T_{m} \cong-\left[J_{m}^{\prime} H_{m}^{\prime \prime}-J_{m}^{\prime \prime} H_{m}^{\prime}\right] k(b-a) .
$$

The argument of the Bessel functions, $J_{m}$ and $H_{m}$, is $k b$, and the double prime indicates a second derivative with respect to $k b$. Making repeated use of the recursion formulas

$$
\begin{gathered}
J_{m+1}^{\prime}=-\frac{m+1}{k b} J_{m+1}+J_{m} \\
J_{m}^{\prime}=\frac{m}{k b} J_{m}-J_{m+1}
\end{gathered}
$$

and utilizing the Wronskian relation

$$
J_{m} H_{m}^{\prime}-H_{m} J_{m}^{\prime}=\frac{2}{\pi i k b}
$$

it is easy to show that

$$
T_{m} \cong \frac{2 i}{\pi}\left(1-\frac{m^{2}}{k^{2} b^{2}}\right)\left(\frac{b-a}{b}\right) .
$$

In a similar manner, an expansion in ascending powers of $k(b-a)$ can be developed for $L_{m}$. Retaining terms to first order in $k(b-a)$ leads to

$$
\begin{aligned}
L_{m} & \simeq \frac{2}{\pi i k b}-\frac{b-a}{b}\left[J_{m} H_{m+1}-J_{m+1} H_{m}\right] \\
& \simeq \frac{2}{\pi i k b}+\frac{b-a}{b}\left[\frac{2}{\pi i k b}\right] \\
& \simeq \frac{2}{\pi i k a} .
\end{aligned}
$$

2 J. R. Wait, Slotted-cylinder antenna with a dielectric coating, Addendum to Radio Physics Lab. Project Report 19-0-11 (Ottawa, November 1954).
On the basis of the above derivation it would be expected that these formulas for $T_{m}$ and $L_{m}$ are valid if $(b-a) \ll b$ and $k(b-a)<1$.

The expression for the pattern factor now takes the relatively simple form

$$
P(\phi) \cong \frac{1}{B} \sum_{m=0}^{\infty} \frac{\epsilon_{m} \cos m \phi e^{i m \pi / 2}}{H_{m}^{\prime}(B)+G_{m} H_{m}(B)}
$$

where

$$
G_{m}=\left(\mu / \mu_{0}\right)(B-A)\left(1-\frac{m^{2}}{N^{2} B^{2}}\right) \frac{A}{B}
$$

with $A=k_{0} a, B=k_{0} b, N=k / k_{0}$. It can be seen that if $k=k_{0}$, or if $b=a$, the equation for the pattern becomes

$$
P(\phi)=\frac{1}{A} \sum_{m=0}^{\infty} \frac{\boldsymbol{\epsilon}_{m} \cos m \phi e^{i m \pi / 2}}{H_{m}^{\prime}(A)}
$$

which is the well-known result for the uncoated cylinder (see footnote 1 ).

\section{Corrugated Cylinder}

Before discussing numerical results, it is of some interest to consider a closely related problem. A line magnetic source (of strength $K$ volts) is located at $\rho=\rho_{0}, \phi=0$, parallel to a circular cylinder of radius $b$ and infinite length (see fig. 2). The ratio between the tangential electric field $\left(E_{\phi}\right)$ and the tangential magnetic field $\left(H_{z}\right)$ on the surface of the cylinder is to be specified. That is,

$$
\left[E_{\phi}=\frac{i}{\epsilon_{0} \omega} \frac{\partial \psi}{\partial \rho}=-Z \psi\right]_{\rho=b}
$$

where $\psi=H_{z}$ and where $Z$ is the boundary impedance. Following the method suggested previously (see footnote 1 ) the solution for $\rho>b$ can be written

$\psi=\frac{\epsilon_{0} \omega K}{4} \sum_{m=0}^{\infty} \epsilon_{m} H_{m}\left(k_{0} \rho_{0}\right)\left[J_{m}\left(k_{0} \rho\right)+A_{m} H_{m}\left(k_{0} \rho\right)\right]$ $\cos m \phi$ for $\rho<\rho_{0}$

and

$\psi=\frac{\epsilon_{0} \omega K}{4} \sum_{m=0}^{\infty} \epsilon_{m} H_{m}\left(k_{0} \rho\right)\left[J_{m}\left(k_{0} \rho_{0}\right)+A_{m} H_{m}\left(k_{0} \rho_{0}\right)\right]$

$$
\cos m \phi \text { for } \rho>\rho_{0}
$$

where $A_{m}$ is to be determined from the boundary condition at $\rho=b$. In the present case it is easily found that

$$
A_{m}=-\left[\frac{J_{m}^{\prime}\left(k_{0} b\right)+G J_{m}\left(k_{0} h\right)}{H_{m}^{\prime}\left(k_{c} b\right)+G H_{m}\left(k_{0} b\right)}\right]
$$

where $G=-i Z / \eta_{0}, \eta_{0}=\sqrt{\mu_{0} / \epsilon_{0}}$.

The above result is now specialized in two ways. Firstly, the far field approximation is made $\left(k_{0} \rho \gg 1\right)$ and, consequently, the leading term of the asymp- 


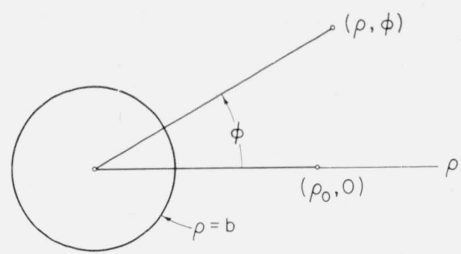

FIgURE 2. Illustrating a line magnetic source at $\left(\rho_{0}, 0\right)$ outside a circular cylinder whose average surface impedance, at $\rho=b$, is to be specified.

totic expansion of $H_{m}\left(k_{0} \rho\right)$ can be used. Secondly, the source is allowed to approach the cylinder $\left(\rho_{0} \rightarrow b\right)$. Then, utilizing the Wronskian for $J_{m}$ and $H_{m}$, the far field can be written

$$
E_{\phi}=-i 60 \epsilon_{0} \omega V\left(\frac{2}{\pi k_{0} \rho}\right)^{1 / 2} e^{-i\left(k_{0} \rho-\pi / 4\right)} Q(\phi)
$$

where the pattern factor $?(\phi)$ is given by

$$
Q(\phi)=\frac{1}{B} \sum_{m=0}^{\infty} \frac{\epsilon_{m} \cos m \phi e^{i m \pi / 2}}{H_{m}^{\prime}(B)+G H_{m}(B)}
$$

where $B=k_{0} b$.

This formula is almost identical in form to that for $P(\phi)$, the pattern of a slotted cylinder with a dielectric coating. The surface impedance function, $Z$, although not strictly applicable to curved surfaces, would be given by

$$
Z \cong i \sqrt{\mu / \epsilon} \tan k(b-a) \cong i \sqrt{\mu / \epsilon} k(b-a),
$$

which is the appropriate value for a thin dielectric film of thickness $b-a$ on a flat perfect conductor for a plane wave at normal incidence. Therefore,

$$
\begin{aligned}
G & \cong \frac{\mu}{\mu_{0}} \cdot \frac{1}{N} \tan N(B-A) \\
& \simeq \frac{\mu}{\mu_{0}}(B-A) .
\end{aligned}
$$

It is interesting to note that

$$
\frac{G_{m}}{G} \simeq\left(1-\frac{m^{2}}{N^{2} B^{2}}\right) \frac{A}{B} \simeq\left(1-\frac{m^{2}}{N^{2} B^{2}}\right) .
$$

If the refractive index, $N$, of the coating is large, the quantity $m^{2} / N^{2} B^{2}$ is small compared to unity for significant values of $m$ (i. e., $m<2 A$ ) in the summation. Consequently, $G_{m} \simeq G$, for thin dielectric coatings of high refractive index. In the case of polystyrene and other such dielectric materials, $N$ is of the order of 2 and the term $m^{2} / N^{2} B^{2}$ is comparable to unity for values of $m$ of the order of $A$. In this case, it is not justified to use an approximate boundary condition. ${ }^{3}$

Although there are severe limitations to the use of approximate boundary conditions for dielectric-clad surfaces, they become very suitable for characterizing corrugated surfaces. For example, if axial grooves of width, $w$, and depth, $h$, are cut circumferentially around the metal cylinder, it follows that the average surface impedance at $\rho=b$ is

$$
Z \cong i \eta_{0}\left(\frac{\mu}{\mu_{0}}\right)\left(\frac{w}{d N}\right) \tan N k_{0} h
$$

where $d$ is the periodicity of the grooves in the circumferential direction, and $N$ is the refractive index of the material filling the grooves. Equation (18a) is obtained by regarding the grooves as shorted transmission lines of electrical length, Nkh, and considering only the fundamental (TEM) mode. This can be expected to be valid ${ }^{4}$ if $d$ is small compared to the wavelength. In the limiting case where $h$ tends to zero, the cylinder again behaves as a perfectly conducting object. When the width of the teeth is small compared to the width of the grooves, such that $w \cong d$, it is seen that

$$
Z \simeq i \eta_{0}\left(\frac{\mu}{\mu_{0}}\right) \frac{1}{N} \tan N k_{0} h
$$

or

$$
G \cong \frac{\mu}{\mu_{0}} \frac{1}{N} \tan N(B-A),
$$

where $Z$ corresponds to the surface impedance for a plane wave at normal incidence on a dielectric film of thickness $b-a$. In the present instance, this always can be expected to be a good approximation since energy flow in the circumferential direction in the dielectric film is not permitted because of the presence of the teeth.

The relative location of the slot and the teeth on the corrugated surface has not been specified. Presumably the slot could be cut in the bottom of one of the grooves.

\section{Numerical Results}

The approximate formula for the pattern function of an axial slot (of finite or infinite length) on a pure dielectric $\left(\mu=\mu_{0}\right)$ clad cylinder is

$$
P(\phi) \cong \frac{1}{B} \sum_{m=0}^{\infty} \frac{\epsilon_{m} \cos m \phi e^{i m \pi / 2}}{H_{m}^{\prime}(B)+G_{m} H_{m}(B)}
$$

3 Some improvement might be obtained if the value of the surface impedance for a plane wave at grazing incidence on a thin dielectric coating on a plane conductor was used. This would lead to

$$
G \cong \frac{\mu}{\mu_{0}}(B-A)\left(1-\frac{1}{N^{2}}\right) .
$$

${ }^{4}$ R. S. Elliott, Azimuthal surface waves on corrugated cylinders, J. Appl. Phys. 38,368 (April, 1955). 
where $G_{m}=(B-A)\left[1-\left(m^{2} / N^{2} B^{2}\right)\right] A / B$. The amplitude and phase of $P(\phi)$ for $\phi$ from $0^{\circ}$ to $180^{\circ}$ are shown listed in tables 1 and 2 . The columns labeled "exact" are based on the exact formula for $P(\phi)$ given by eq (2), and the columns labeled "approx." are based on eq (19). The amplitude values shown are normalized to unity at $\phi=0$ and the phase, which is -arg. $P(\phi)$, is normalized to $0^{\circ}$ at $\phi=0$.

The cases for the smaller cylinders $(A=2$ and 3$)$ are treated by both the approximate and exact techniques. The excellence of the approximate formula is evidenced by a comparison of the appropriate columns. For the case of the large cylinder $(A=8)$, only the numerical results from the approximate formula have been obtained. The corresponding"exact numerical data for $A=8$ could be obtained only from extensive and tedious computations. It is believed, however, that the approximate results for this larger cylinder would not differ more than 5 percent in amplitude and $10^{\circ}$ in phase from reality.

The corresponding formula for $Q(\phi)$, the pattern of a slot on a corrugated cylinder, is identical in form to that for $P(\phi)$, except $G_{m}$ is replaced by $G$ where

$$
G=\frac{1}{N} \tan [N(B-A)] .
$$

In this case, the teeth are of small width compared to their separation. The refractive index of the dielectric material filling the grooves is $N$ and the depth of the grooves is $b-a$. One might describe this situation as a dielectric-clad cylinder with radial fins contained within the dielectric coating (see fig. $3)$. For purposes of comparison with the noncorrugated cylinder, $Q(\phi)$ has been calculated for the same range of values of $A, B$, and $N$. Results for

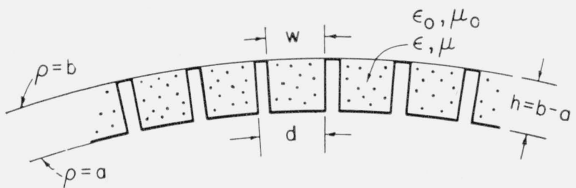

Figure 3. Sectional view of the corrugated surface.

Table 1. Amplitude of $P(\phi)$

\begin{tabular}{|c|c|c|c|c|c|c|c|c|c|c|c|c|c|c|c|}
\hline$\phi$ & $\begin{array}{l}A=2.0 \\
B=2.0\end{array}$ & $\begin{array}{l}A=2.0 \\
B=2.1 \\
\text { Exact } \\
N=2\end{array}$ & $\begin{array}{c}\begin{array}{c}A=2.0 \\
B=2.1 \\
\text { Approx. } \\
N=2\end{array}\end{array}$ & $\begin{array}{l}A=2.0 \\
B=2.2 \\
\text { Exact } \\
N=2\end{array}$ & $\begin{array}{c}A=2.0 \\
B=2.2 \\
\text { Approx. } \\
N=2\end{array}$ & $\begin{array}{l}A=2.0 \\
B=2.3 \\
\text { Exact } \\
N=2\end{array}$ & $\begin{array}{c}A=2.0 \\
B=2.3 \\
\text { Approx. } \\
N=2\end{array}$ & $\begin{array}{l}A=2.0 \\
B=2.2 \\
\text { Exact } \\
N=1.5\end{array}$ & $\begin{array}{c}A=2.0 \\
B=2.2 \\
\text { Approx. } \\
N=1.5\end{array}$ & $\begin{array}{l}A=3.0 \\
B=3.0\end{array}$ & $\begin{array}{l}A=3.0 \\
B=3.2 \\
\text { Exact } \\
N=2\end{array}$ & $\begin{array}{c}A=3.0 \\
B=3.2 \\
\text { Approx. } \\
N=2\end{array}$ & $\begin{array}{l}A=8.0 \\
B=8.0\end{array}$ & $\begin{array}{c}A=8.0 \\
B=8.1 \\
\text { Approx. } \\
N=2\end{array}$ & $\begin{array}{c}A=8.0 \\
B=8.2 \\
\text { Approx. } \\
N=2\end{array}$ \\
\hline $0^{\circ}$ & 1. 000 & 1. 000 & 1.000 & 1.000 & 1.000 & 1.000 & 1.000 & 1. 000 & 1.000 & 1.000 & 1.000 & 1.000 & 1.000 & 1.000 & \\
\hline $10^{\circ}$ & 1.000 & $\begin{array}{l}0.999 \\
0.09\end{array}$ & 1.000 & 1.002 & 0.995 & 1.003 & 1.002 & 1.001 & 0.996 & 0.997 & 1.003 & 1.004 & 0.997 & 0.995 & $\begin{array}{l}1.000 \\
0.998\end{array}$ \\
\hline $20^{\circ}$ & 0.990 & .995 & 0.997 & 1.005 & .987 & 1.012 & 1.012 & 1. 001 & .986 & .987 & 1. 007 & 1.014 & .992 & $\begin{array}{l}.988 \\
\end{array}$ & $\begin{array}{r}.930 \\
.985\end{array}$ \\
\hline $30^{\circ}$ & .976 & .985 & .988 & 1. 005 & .979 & 1. 021 & 1. 024 & 0.995 & .974 & .966 & 1. 002 & 1.020 & .986 & .988 & .990 \\
\hline $40^{\circ}$ & .942 & .961 & .967 & 0.993 & .971 & 1.021 & 1.031 & .975 & .961 & .934 & 0.974 & 1.004 & .966 & .972 & .988 \\
\hline $50^{\circ}$ & .900 & .920 & .927 & .957 & .950 & 0.995 & 1.081 & .936 & .934 & .895 & .924 & 0.955 & .945 & .953 & $\begin{array}{l}.957 \\
.957\end{array}$ \\
\hline $60^{\circ}$ & 838 & .861 & .868 & .894 & .899 & .932 & 0.971 & .876 & .881 & .860 & .874 & .901 & .905 & .928 & $\begin{array}{l}.957 \\
.957\end{array}$ \\
\hline $70^{\circ}$ & .780 & .796 & .800 & .810 & .813 & .836 & .847 & .803 & .801 & .828 & .853 & .874 & .845 & .866 & .887 \\
\hline $80^{\circ}$ & .732 & .742 & .743 & .733 & .722 & .739 & .797 & .738 & .719 & .779 & .842 & .866 & .788 & $\begin{array}{l}.800 \\
.822\end{array}$ & $\begin{array}{l}.807 \\
.869\end{array}$ \\
\hline $90^{\circ}$ & .698 & .711 & .711 & .693 & .674 & 691 & .739 & .703 & .676 & .693 & .784 & .814 & .680 & .716 & $\begin{array}{l}.709 \\
.753\end{array}$ \\
\hline $100^{\circ}$ & .0980 & .695 & .695 & .689 & .678 & .706 & .742 & .694 & .677 & .585 & .657 & $\begin{array}{l}.017 \\
.688\end{array}$ & .601 & .659 & $\begin{array}{l}.724 \\
.724\end{array}$ \\
\hline $110^{\circ}$ & .622 & .661 & .664 & .680 & .692 & .729 & .760 & .673 & .674 & .509 & .539 & .569 & .479 & .522 & .568 \\
\hline $120^{\circ}$ & .536 & .582 & .587 & .620 & .641 & .692 & .719 & .603 & .616 & .490 & .535 & .548 & .401 & .486 & .561 \\
\hline $130^{\circ}$ & $\begin{array}{l}.412 \\
.412\end{array}$ & .454 & .459 & .493 & .508 & .563 & .589 & .473 & .484 & .463 & .562 & .579 & .295 & $\begin{array}{l}400 \\
.333\end{array}$ & $\begin{array}{l}.001 \\
.375\end{array}$ \\
\hline $140^{\circ}$ & .282 & .305 & .308 & .327 & .323 & .366 & .391 & .316 & .310 & .367 & .483 & .502 & .282 & .348 & .428 \\
\hline $150^{\circ}$ & .227 & .232 & .231 & .238 & .215 & .223 & .245 & .23 & .215 & .215 & .282 & .300 & .15 & .179 & .208 \\
\hline $160^{\circ}$ & .285 & .305 & .303 & .331 & .321 & .331 & .343 & .30 & .31 & .162 & .177 & .181 & .20 & .286 & .348 \\
\hline $170^{\circ}$ & .361 & .402 & .401 & .451 & .453 & .485 & .496 & .413 & .432 & .271 & .350 & .335 & .047 & .056 & .066 \\
\hline $180^{\circ}$ & .392 & .441 & .440 & .499 & .499 & .546 & .557 & .454 & .478 & .325 & .436 & .449 & .182 & .265 & .323 \\
\hline
\end{tabular}

TABle 2. Normalized phase of $P(\phi)$

\begin{tabular}{|c|c|c|c|c|c|c|c|c|c|c|c|c|c|c|c|}
\hline$\phi$ & $\begin{array}{l}A=2.0 \\
B=2.0\end{array}$ & $\begin{array}{l}A=2.0 \\
B=2.1 \\
\text { Exact } \\
N=2\end{array}$ & $\begin{array}{c}A=2.0 \\
B=2.1 \\
\text { Approx. } \\
N=2\end{array}$ & $\begin{array}{l}A=2.0 \\
B=2.2 \\
\text { Exact } \\
N=2\end{array}$ & $\begin{array}{c}A=2.0 \\
B=2.2 \\
\text { Approx. } \\
N=20\end{array}$ & $\begin{array}{l}A=2.0 \\
B=2.3 \\
\text { Exact } \\
N=2\end{array}$ & $\begin{array}{c}A=2.0 \\
B=2.3 \\
\text { Approx. } \\
N=2\end{array}$ & $\begin{array}{l}A=2.0 \\
B=2.2 \\
\text { Exact } \\
N=1.5\end{array}$ & $\begin{array}{c}A=2.0 \\
B=2.2 \\
\text { Approx. } \\
N=1.5\end{array}$ & $\begin{array}{l}A=3.0 \\
B=3.0\end{array}$ & $\begin{array}{l}A=3.0 \\
B=3.2 \\
\text { Exact } \\
N=2\end{array}$ & $\begin{array}{c}A=3.0 \\
B=3.2 \\
\text { Approx. } \\
N=2\end{array}$ & $\begin{array}{l}A=8.0 \\
B=8.0\end{array}$ & $\begin{array}{c}A=8.0 \\
B=8.1 \\
\text { Approx. } \\
N=2\end{array}$ & $\begin{array}{c}A=8.0 \\
B=8.2 \\
\text { Approx. } \\
N=2\end{array}$ \\
\hline $0^{\circ}$ & $0^{\circ}$ & $0^{\circ}$ & $0^{\circ}$ & $0^{\circ}$ & $0^{\circ}$ & $0^{\circ}$ & $0^{\circ}$ & $0^{\circ}$ & $0^{\circ}$ & $0^{\circ}$ & $0^{\circ}$ & $0^{\circ}$ & $0^{\circ}$ & $0^{\circ}$ & $0^{\circ}$ \\
\hline $10^{\circ}$ & 1.6 & 1. 6 & 1. 7 & 1.8 & 2.1 & 2.0 & 2.1 & 1.6 & 2.1 & 2.4 & 2.6 & 2.8 & 7.9 & 6.9 & 6.2 \\
\hline $20^{\circ}$ & 6. 2 & 6.6 & 5.0 & 6.9 & 8.4 & 7.6 & 8.3 & 6.5 & 8.0 & 9.6 & 10.1 & 10.3 & 27.5 & 27.8 & 27.0 \\
\hline $30^{\circ}$ & 13.9 & 14.5 & 15.0 & 15.1 & 18.3 & 16.3 & 17.8 & 14.5 & 17. 3 & 21.5 & 22.3 & 23.3 & 60.7 & 61.6 & 62.3 \\
\hline $40^{\circ}$ & 24.4 & 25.4 & 26.4 & 26.0 & 3.6 & 27.6 & 30.0 & 25.4 & 29. 3 & 38.2 & 39.1 & 40. 1 & 105. 8 & 107.1 & 108.0 \\
\hline $50^{\circ}$ & 37.9 & 39. 2 & 39.8 & 39.5 & 45.0 & 41.0 & 44. 4 & 39.2 & 43. 2 & 59.6 & 61.1 & 61.7 & 161.8 & 164.5 & 166.8 \\
\hline $60^{\circ}$ & 54.6 & 56.1 & 61.8 & 55. 9 & 60.5 & 57.2 & 61.3 & 56.0 & 59.3 & 85.0 & 88.7 & 89.6 & 226.1 & 229.6 & 233.1 \\
\hline $70^{\circ}$ & 74.4 & 76.5 & 82.9 & 76.2 & 79.6 & 77.2 & 84.3 & 76.3 & 78.5 & 112.5 & 119.9 & 121.3 & 298.9 & 333.7 & 307.9 \\
\hline $80^{\circ}$ & 96.8 & 100.1 & 107.3 & 100.9 & 103. 7 & 102.7 & 106. 2 & 100.3 & 102.3 & 141.1 & 150.9 & 138.0 & 374. 9 & 382.5 & 388.8 \\
\hline $90^{\circ}$ & 120.1 & 125.1 & 132.1 & 128.6 & 132.3 & 132.9 & 136. 4 & 126.4 & 129.7 & 171.2 & 187. 9 & 184.4 & 459. 6 & 466.6 & 473.2 \\
\hline $100^{\circ}$ & 142.4 & 148. 9 & 156.1 & 155.1 & 160.1 & 162.1 & 166.1 & 151.3 & 151.2 & 205.4 & 214.0 & 216.3 & 543.8 & 552.9 & 562.5 \\
\hline $110^{\circ}$ & 162.6 & 169.9 & 176.1 & 177.8 & 181.9 & 185.7 & 188.7 & 172.8 & 177.5 & 244. & 256.4 & 254.7 & 632.3 & 641.8 & 650.7 \\
\hline $120^{\circ}$ & 181.6 & 189.0 & 194.6 & 197.1 & 199. 3 & 204.5 & 210.8 & 191.7 & 195.0 & 282. & 371.6 & 304.1 & 718.1 & 730.9 & 744.5 \\
\hline $130^{\circ}$ & 202.0 & 208.8 & 213.3 & 216.4 & 216.1 & 221.8 & 229.2 & 211.0 & 212.2 & 312.7 & 334.2 & 337.1 & 804.0 & 821.4 & 832.5 \\
\hline $140^{\circ}$ & 231.2 & 237.1 & 238.3 & 243.7 & 243.7 & 245.3 & 253.3 & 238.7 & 237.7 & 337.6 & 357.9 & 361.5 & 894.2 & 910.7 & 928.7 \\
\hline $150^{\circ}$ & 278.4 & 287.4 & 281.5 & 296.9 & 297.2 & 300.3 & 306.0 & 289.8 & 292.8 & 371.1 & 386.4 & 389.1 & 988. 4 & 1001.8 & 1014.4 \\
\hline $160^{\circ}$ & 317.8 & 330.1 & 322.9 & 342.9 & 346.3 & 356.5 & 361.5 & 335.0 & 339.9 & 445.3 & 469.7 & 472.6 & 1089.1 & 1090.4 & $\begin{array}{l}1112.4 \\
\end{array}$ \\
\hline $170^{\circ}$ & 334.6 & 347.2 & 342.2 & 359.7 & 362.0 & 374.0 & 379.9 & 352.7 & 355.6 & 485.1 & 514. 6 & 518.5 & 1168.1 & $\begin{array}{l}1179.4 \\
\end{array}$ & 1195.5 \\
\hline $180^{\circ}$ & 339.1 & 351.6 & 347.6 & 364.0 & 366.6 & 378.1 & 384.5 & 357.3 & 359.5 & 492.8 & 521.8 & 525.8 & 1249.3 & 1270.3 & 1293. 9 \\
\hline
\end{tabular}


the normalized amplitude and phase of $Q(\phi)$ are listed in tables 3 and 4.

TABLE 3. Amplitude of $Q(\phi)$

\begin{tabular}{|c|c|c|c|c|c|c|c|}
\hline$\phi$ & $\begin{array}{l}A=2.0 \\
B=2.0\end{array}$ & $\begin{array}{l}A=2.0 \\
B=2.1 \\
N=2\end{array}$ & $\begin{aligned} A & =2.0 \\
B & =2.2 \\
N & =2\end{aligned}$ & $\begin{array}{l}A=2.0 \\
B=2.3 \\
N=2\end{array}$ & $\begin{array}{l}A=8.0 \\
B=8.0\end{array}$ & $\begin{array}{l}A=8.0 \\
B=8.1 \\
N=2\end{array}$ & $\begin{array}{l}A=8.0 \\
B=8.2 \\
N=2\end{array}$ \\
\hline $\begin{array}{c}0^{\circ} \\
10^{\circ} \\
20^{\circ} \\
30^{\circ} \\
40^{\circ} \\
50^{\circ} \\
60^{\circ} \\
70^{\circ} \\
80^{\circ} \\
90^{\circ} \\
100^{\circ} \\
110^{\circ} \\
120^{\circ} \\
130^{\circ} \\
140^{\circ} \\
150^{\circ} \\
157.5^{\circ} \\
160^{\circ} \\
170^{\circ} \\
180^{\circ}\end{array}$ & $\begin{array}{r}1.000 \\
1.000 \\
0.990 \\
.976 \\
.942 \\
.900 \\
.838 \\
.780 \\
.732 \\
.698 \\
.670 \\
.622 \\
.536 \\
.412 \\
.282 \\
.227 \\
-.285 \\
.361 \\
.392\end{array}$ & $\begin{array}{r}1.000 \\
1.001 \\
1.001 \\
0.996 \\
.978 \\
.941 \\
.882 \\
.811 \\
.748 \\
.718 \\
.707 \\
.683 \\
.610 \\
.480 \\
.320 \\
.234 \\
-.312 \\
.419 \\
.462\end{array}$ & $\begin{array}{r}1.000 \\
0.995 \\
.996 \\
1.006 \\
1.023 \\
1.026 \\
0.983 \\
.886 \\
.768 \\
.708 \\
.737 \\
.783 \\
.754 \\
.618 \\
.412 \\
.282 \\
.390 \\
.541 \\
.600\end{array}$ & $\begin{array}{r}1.000 \\
1.000 \\
1.006 \\
1.027 \\
1.062 \\
1.089 \\
1.072 \\
0.988 \\
.856 \\
.757 \\
.776 \\
.859 \\
.879 \\
.759 \\
.507 \\
.265 \\
-.394 \\
.624 \\
.713\end{array}$ & $\begin{array}{r}1.000 \\
0.997 \\
.992 \\
.986 \\
.966 \\
.945 \\
.905 \\
.845 \\
.788 \\
.680 \\
.601 \\
.179 \\
.401 \\
.295 \\
.282 \\
.155 \\
.205 \\
.205 \\
.047 \\
.182\end{array}$ & $\begin{array}{r}1.000 \\
0.991 \\
.986 \\
1.005 \\
1.000 \\
0.976 \\
.949 \\
.905 \\
.883 \\
.749 \\
.669 \\
.553 \\
.538 \\
.356 \\
.391 \\
.193 \\
.268 \\
.285 \\
.083 \\
.309\end{array}$ & $\begin{array}{r}1.000 \\
1.007 \\
0.999 \\
.996 \\
1.005 \\
0.987 \\
.985 \\
.921 \\
.908 \\
.795 \\
.781 \\
.652 \\
.635 \\
.424 \\
.513 \\
.241 \\
.400 \\
.439 \\
.088 \\
.415\end{array}$ \\
\hline
\end{tabular}

TABLE 4. Normalized phase of $Q(\phi)$

\begin{tabular}{|c|c|c|c|c|c|c|c|}
\hline$\phi$ & $\begin{array}{l}A=2.0 \\
B=2.0\end{array}$ & $\begin{aligned} A & =2.0 \\
B & =2.1 \\
N & =2\end{aligned}$ & $\begin{aligned} A & =2.0 \\
B & =2.2 \\
N & =2\end{aligned}$ & $\begin{aligned} A & =2.0 \\
B & =2.3 \\
N & =2\end{aligned}$ & $\begin{array}{l}A=8.0 \\
B=8.0\end{array}$ & $\begin{aligned} A & =8.0 \\
B & =8.1 \\
N & =2\end{aligned}$ & $\begin{array}{l}A=8.0 \\
B=8.2 \\
N=2\end{array}$ \\
\hline $0^{\circ}$ & $0^{\circ}$ & $0^{\circ}$ & $0^{\circ}$ & $0^{\circ}$ & $0^{\circ}$ & $0^{\circ}$ & $0^{\circ}$ \\
\hline $10^{\circ}$ & 1. 6 & 8.6 & 12.5 & 7.8 & 7.9 & 7.0 & 6.2 \\
\hline $20^{\circ}$ & 6.2 & 13.5 & 19. 9 & 15.7 & 27.5 & 28.5 & 26.6 \\
\hline $30^{\circ}$ & 13.9 & 20.3 & 30.8 & 27.7 & 60.7 & 62.8 & 63.0 \\
\hline $40^{\circ}$ & 24.4 & 32.4 & 43.7 & 42.1 & 105.8 & 108.1 & 109.6 \\
\hline $50^{\circ}$ & 37.9 & 46.1 & 57.6 & 57.6 & 161.8 & 165.7 & 168.1 \\
\hline $60^{\circ}$ & 54.6 & 62.9 & 72.8 & 74.2 & 226.1 & 231.8 & 237.1 \\
\hline $70^{\circ}$ & 74.4 & 83.4 & 91.2 & 93.3 & 298.9 & 307.0 & 310.9 \\
\hline $80^{\circ}$ & 96.8 & 107.5 & 115.7 & 117.9 & 374.9 & 384.7 & 395.7 \\
\hline $90^{\circ}$ & 120.1 & 133.6 & 146.8 & 150.6 & 459.6 & 467.1 & 478.1 \\
\hline $100^{\circ}$ & 142.4 & 158.4 & 176.9 & 178.3 & 543.8 & 556.8 & 572.5 \\
\hline $110^{\circ}$ & 162.6 & 170.2 & 200.0 & 213.7 & 632.3 & 647.5 & 657.6 \\
\hline $120^{\circ}$ & 181.6 & 199.1 & 217.5 & 233.8 & 718.1 & 735.8 & 757.8 \\
\hline $130^{\circ}$ & 202.0 & 209.5 & 234.3 & 250.0 & 804.0 & 824.5 & 841,1 \\
\hline $140^{\circ}$ & 231.2 & 245.9 & 258.0 & 270.0 & 894.2 & 917.1 & 945.0 \\
\hline $150^{\circ}$ & 278.4 & 297.0 & 309.1 & 302.3 & 988.4 & 1001.7 & 1024.3 \\
\hline $160^{\circ}$ & 317. 8 & 341.1 & 357.3 & 390.0 & $\begin{array}{r}1089.1 \\
\end{array}$ & 1100.3 & 1130.8 \\
\hline $170^{\circ}$ & $\begin{array}{l}334.6 \\
0\end{array}$ & 358.5 & 374.3 & 408. 8 & 1168.1 & 1204.6 & 1206.1 \\
\hline $180^{\circ}$ & 339.1 & 364.0 & 378.4 & 412.8 & 1249.3 & 1277.5 & 1312.9 \\
\hline
\end{tabular}

\section{Graphical Presentation of Results}

The salient features of the radiation patterns are best illustrated by graphical presentation. In figure 4a, $|P(\phi)|$, using the exact formula, is plotted for $A=2.0$, and $B=2.0,2.1,2.2,2.3$ with $N=2.0$. This shows clearly the effect of varying the thickness of the dielectric film. The corresponding results for $|P(\phi)|$, using the approximate formula, are shown in figure $4 \mathrm{~b}$. The two sets of patterns are identical in appearance. The most significant feature is that the dielectric film enhances the field to the rear. The pattern $|Q(\phi)|$ for the corrugated cylinder for the same values of $A, B$, and $N$ is shown in figure $4 \mathrm{c}$. There is a general similarity between these and the corresponding patterns for the uncorrugated dielectric film (i. e., fig. $4 \mathrm{a}$ or $4 \mathrm{~b}$ ). It appears that the corrugations further enhance the field in the backward direction.
The effect of varying $N$, the refractive index of the dielectric coating, is shown in figures $5 \mathrm{a}$ and $5 \mathrm{~b}$ where $|P(\phi)|$ is plotted for $A=2.0, B=2.2$, and $N=$ 1.5 and 2.0. As would be expected, the distortion of the pattern for $N=1.5$ from the uncoated case (i. e., $A=B=2.0$ ) is less than for the higher value of $N$. The corresponding patterns for $Q(\phi)$ and $N=1.5$ also have less distortion than that for $N=$ 2.0 , which was plotted in figure $4 \mathrm{c}$. This is readily evident from the formula

$$
G=\frac{1}{N} \tan N(B-A) \cong(B-A)+\frac{N^{2}(B-A)^{3}}{3}+\cdots
$$

which to a first order in $(B-A)$ is independent of $N$, but tends to increase with $N$ when $B-A$ is not small.

The patterns, $P(\phi)$, for a slightly larger cylinder, $(A=3.0)$, are shown in figures $6 \mathrm{a}$ and $6 \mathrm{~b}$ using the exact and approximation formulas, respectively. The rear lobes are now becoming smaller. The enhancement, resulting from the presence of the coating of the field in the backward direction, is again quite significant.

Finally, the pattern $|P(\phi)|$ for the large dielectriccoated cylinder is shown in figure 7 a for several thicknesses of the coating. The corresponding pattern $|Q(\phi)|$ for the corrugated cylinder is shown in figure $7 \mathrm{~b}$. It is strikingly noted here that the pattern for the corrugated cylinder has a pronounced lobe structure. This is evidence that surface waves are being guided more efficiently, due to the presence of the corrugations. This viewpoint is further substantiated when eq (19) is transformed to a Watson-Bremmer type ${ }^{5}$ residue series. The individual terms of this mode series are then in the form of peripheral surface waves. The dominant mode is of the type studied by Elliott (see footnote 3).

\section{Conclusion}

It is seen that considerable simplification can be made to the general formula for radiation from a dielectric-coated, axially slotted cylinder. This new approximate formula, which is valid for thin coatings, is only slightly more complicated than its counterpart for an uncoated cylinder. It is shown that a direct approach, using approximate boundary conditions, is not generally valid for curved dielectricclad surfaces due to the azimuthal flow of energy within the dielectric. On the other hand, the formulas derived using the surface impedance concept are applicable to a corrugated surface, and the patterns calculated therefrom have similar characteristics to their dielectric-clad counterpart. Generally speaking, for a specified normal surface impedance, the corrugated cylinder modifies the pattern to a greater extent than the dielectric-clad cylinder.

${ }^{5} \mathrm{H}$. Bremmer, Terrestrial radio waves (Elsevier Publishing Company, New York, N. Y., 1949). 


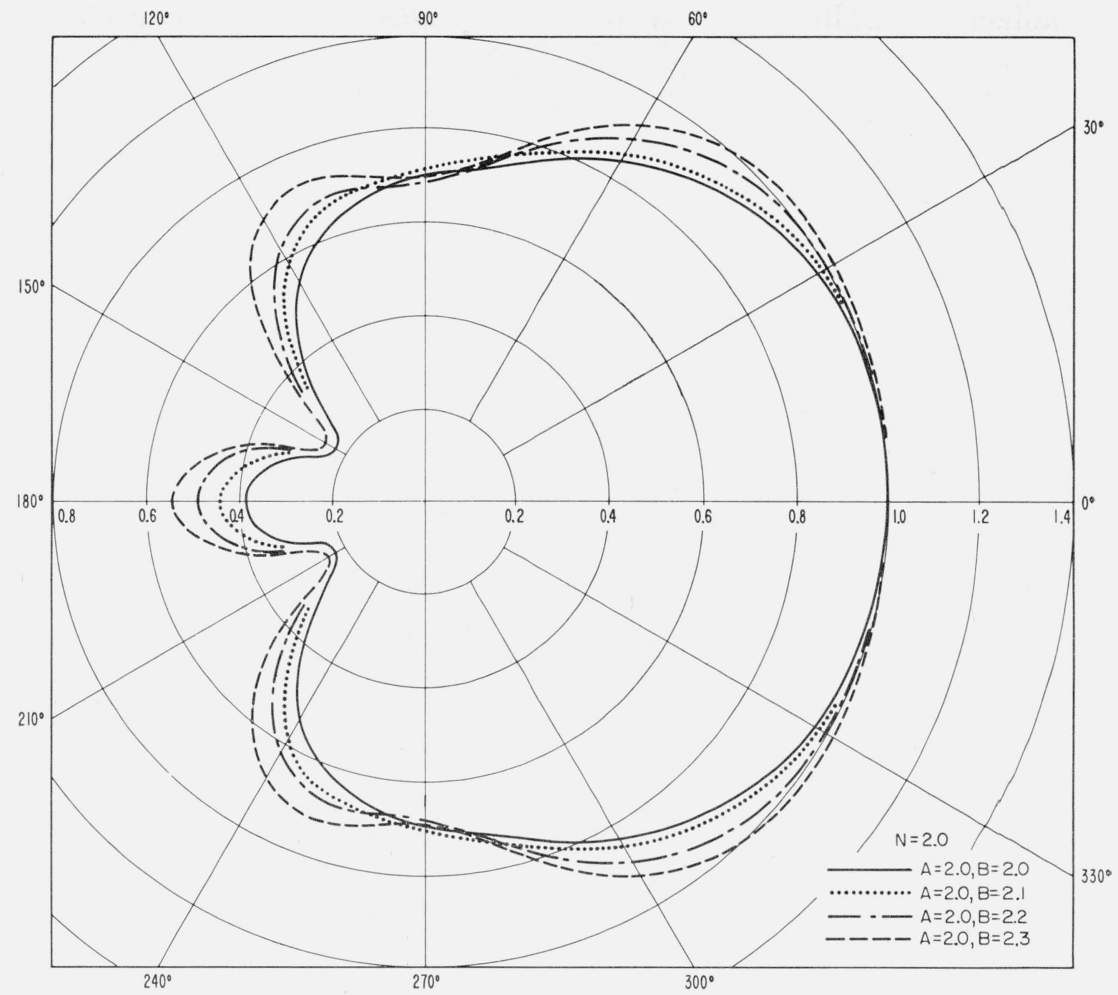

Figure 4a. Pattern $|P(\phi)|$ (exact).

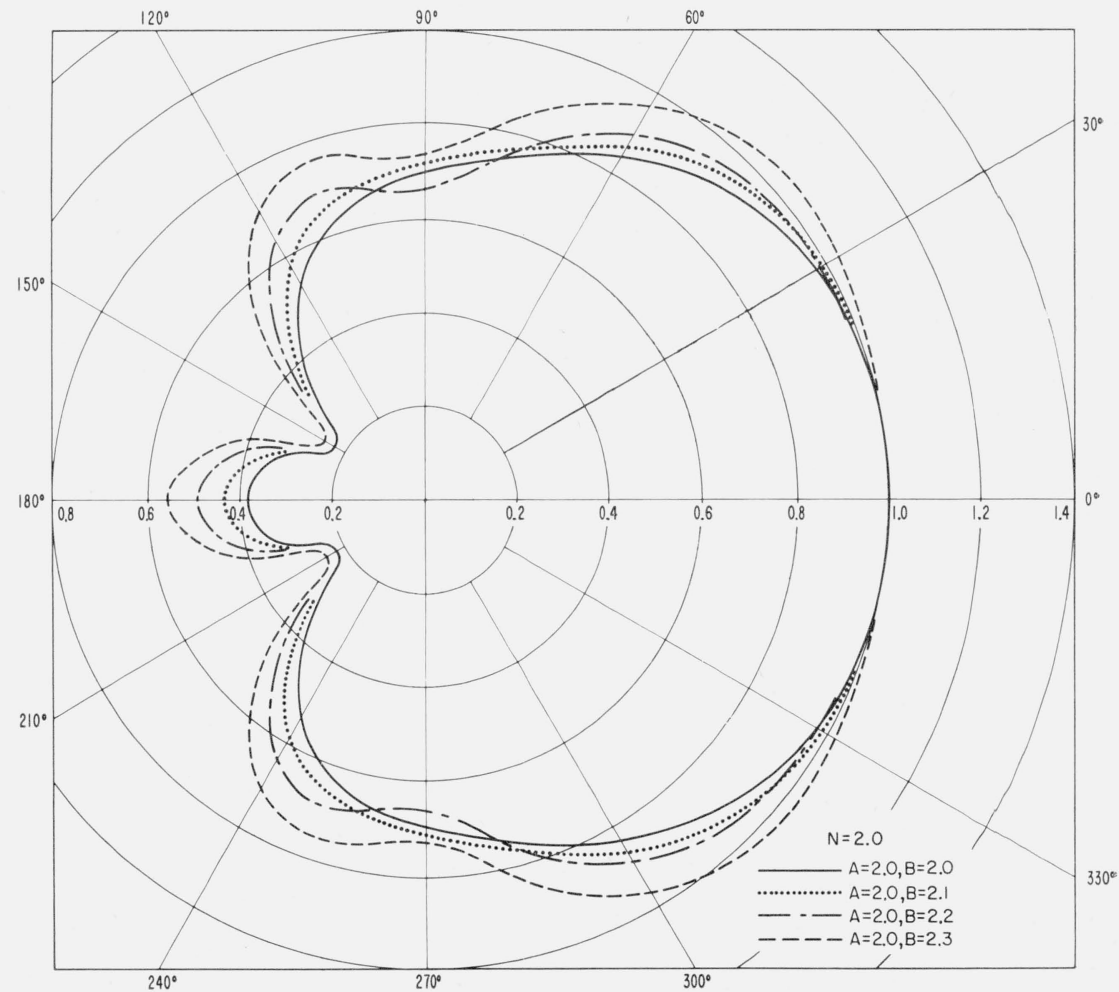

Figure 4b. Pattern $|P(\phi)|$ (approximate). 


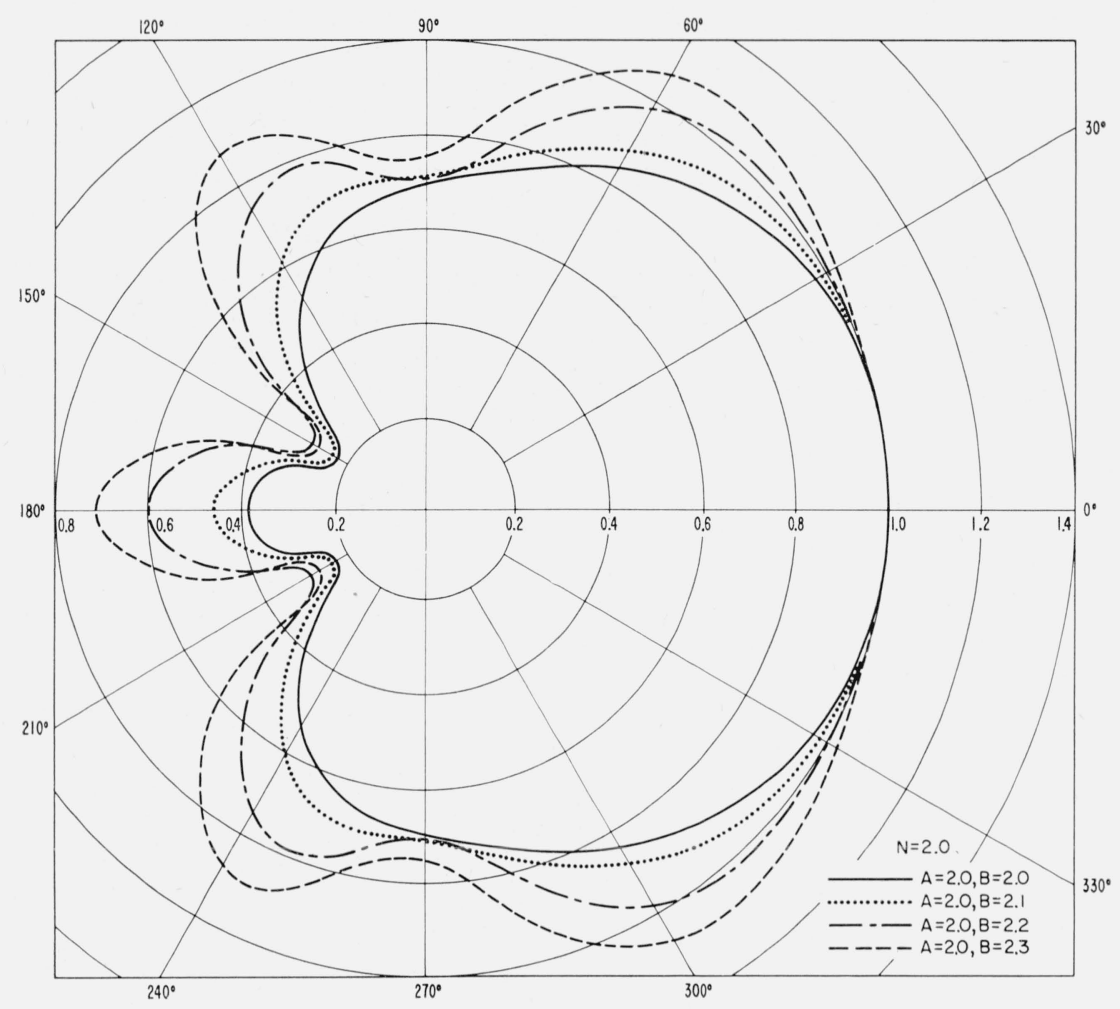

Figure 4c. Pattern $|Q(\phi)|$.

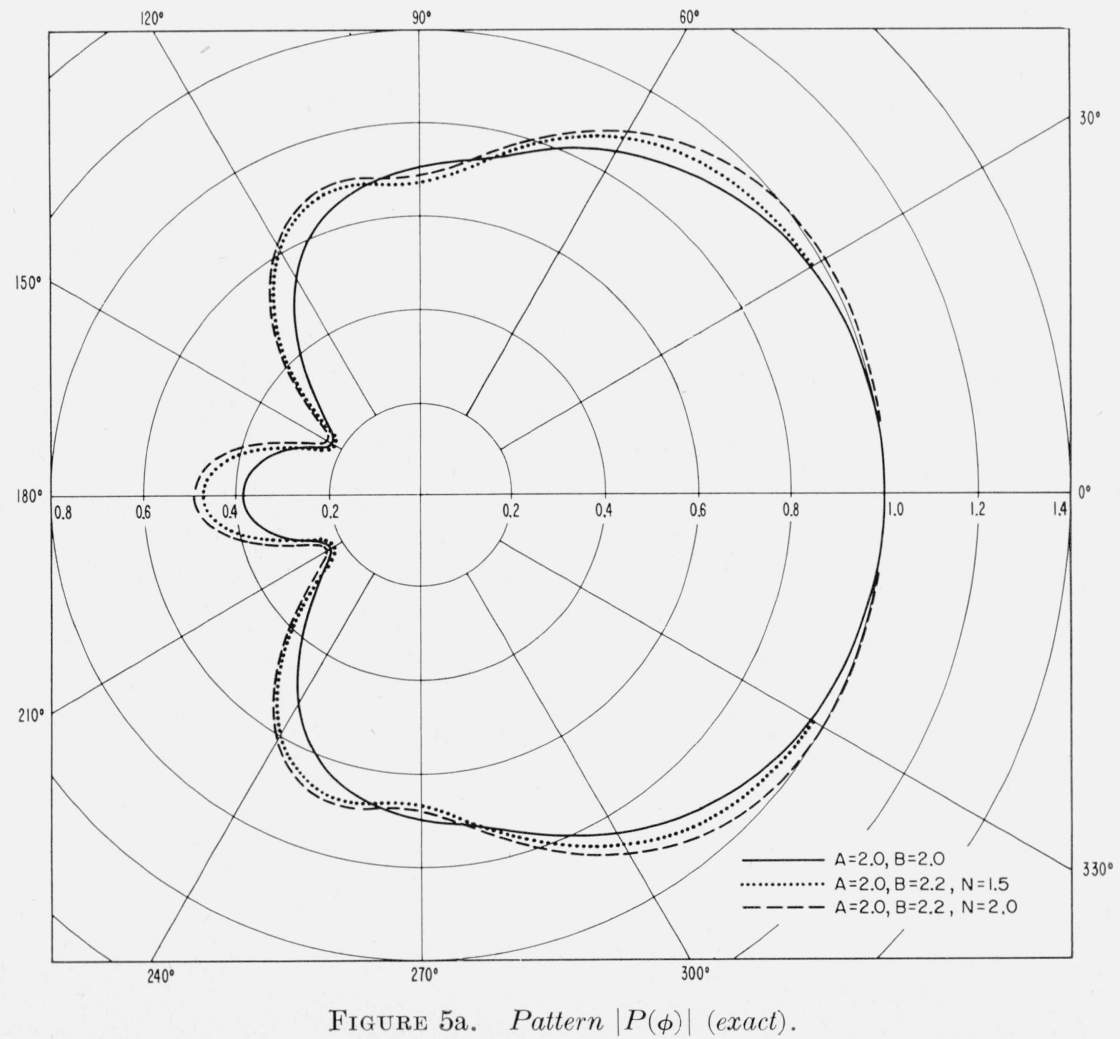




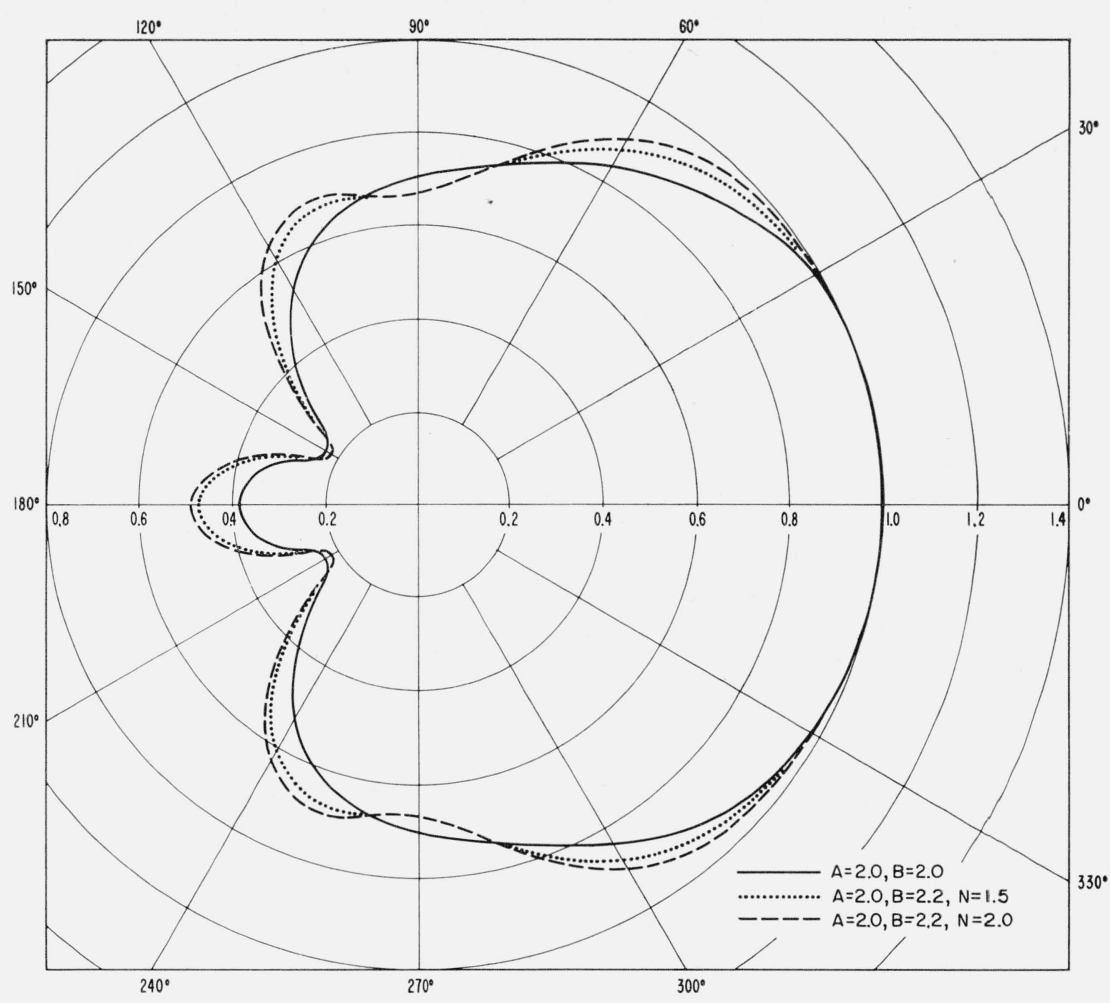

Figure 5b. Pattern $|P(\phi)|$ (approximate).

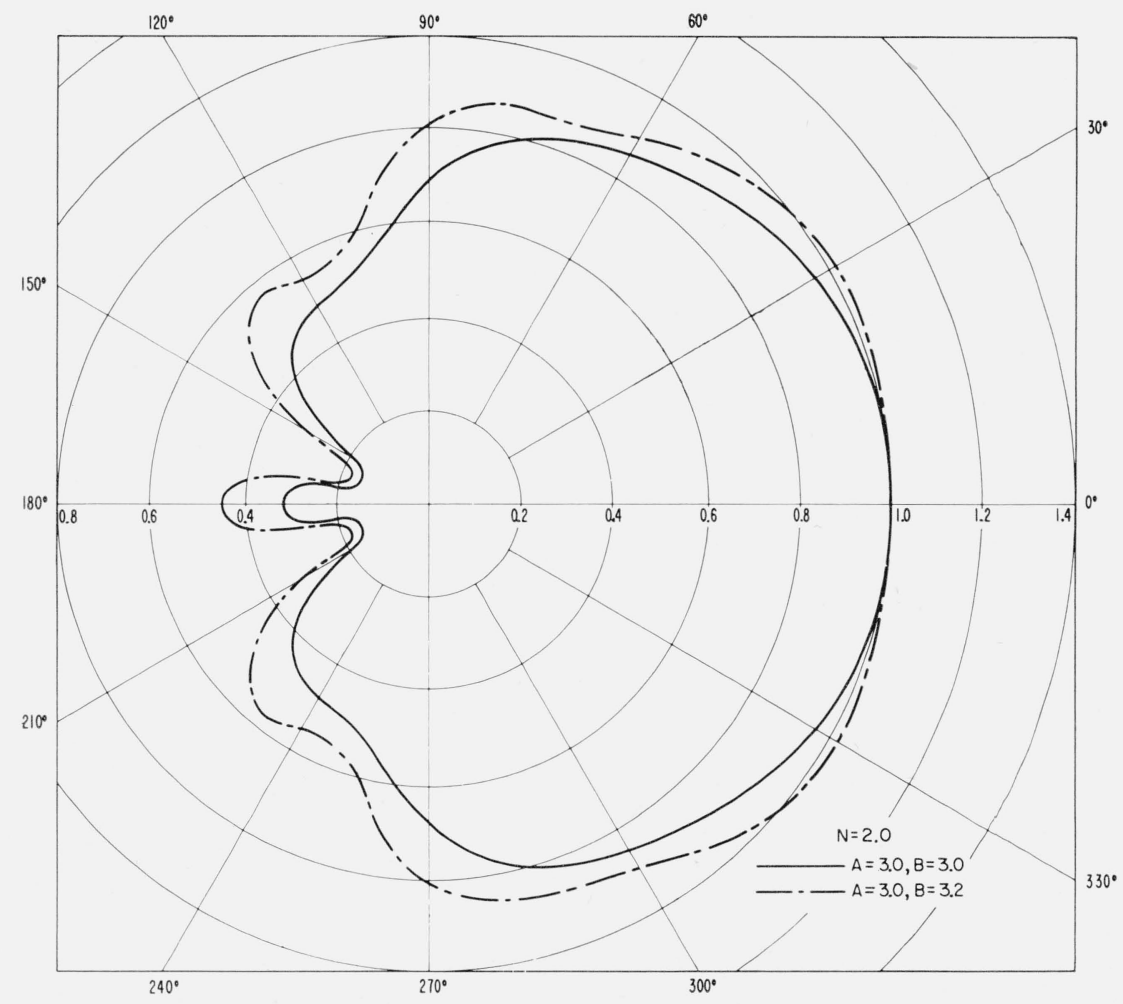

Figure 6a. Pattern $|P(\phi)|$ (exact). 


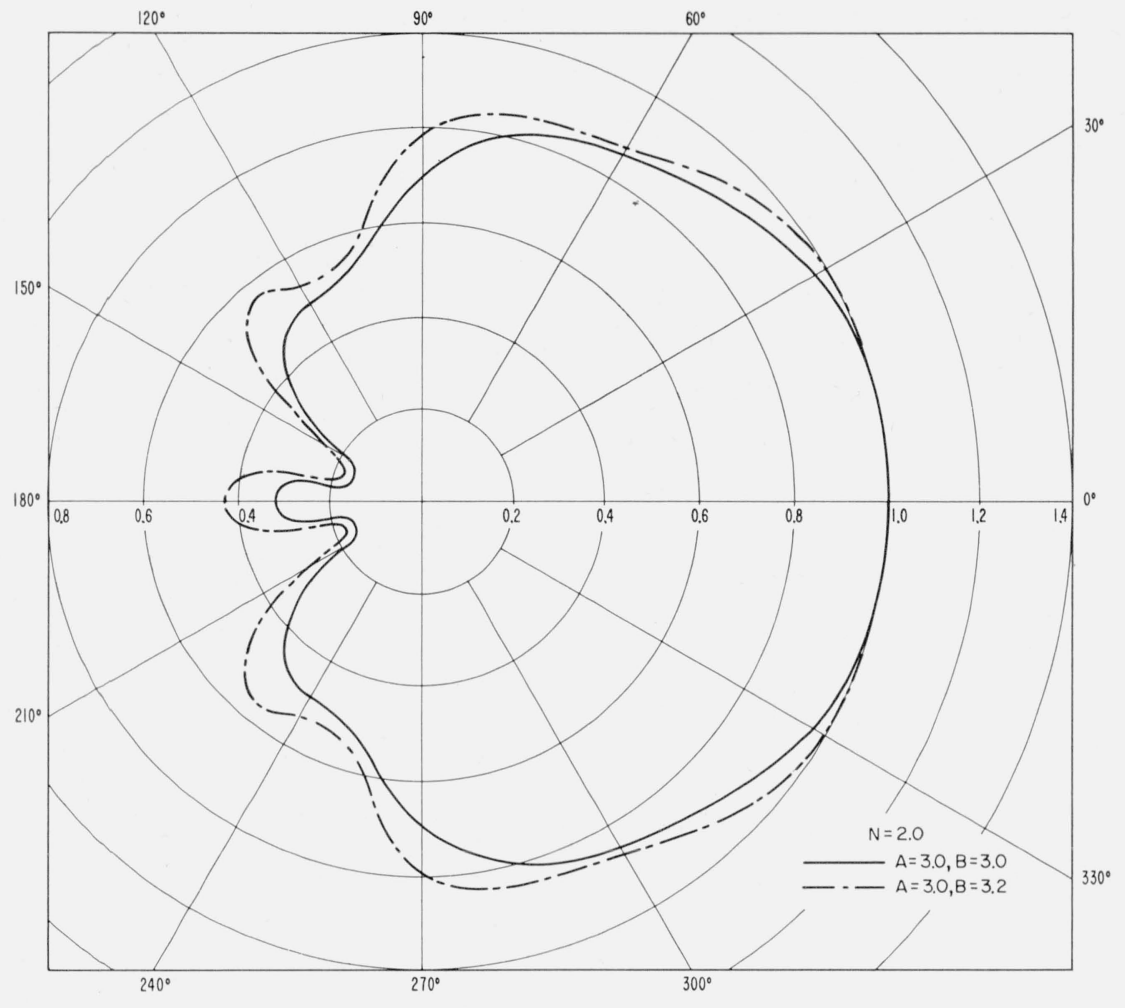

Figure 6b. Pattern $|P(\phi)|$ (approximate).

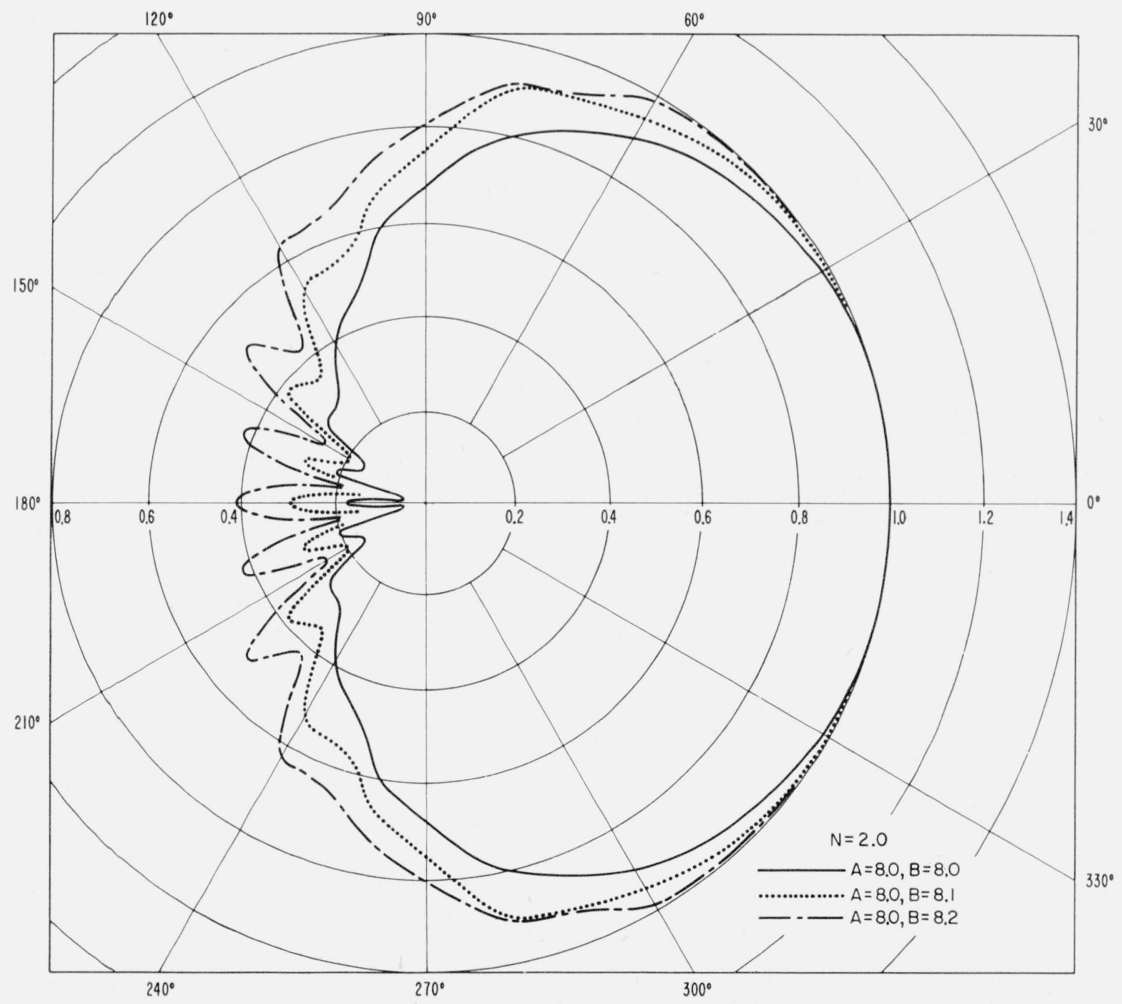

Figure 7a. Pattern $|P(\phi)|$ (approximate). 


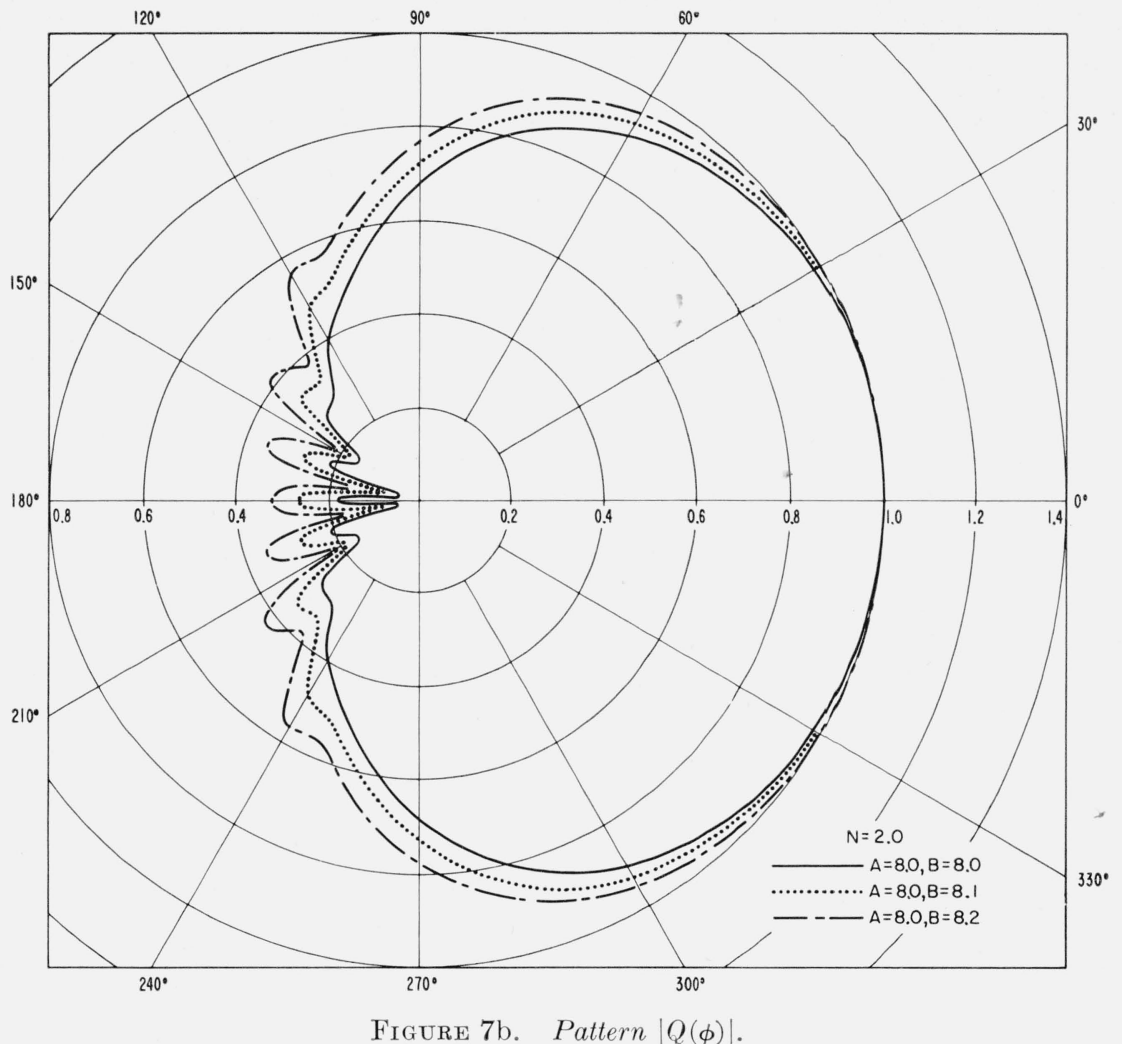

Boulder, Colo., May 9, 1957. 